\title{
The DLR C-Runner: Concept, Design and Experiments
}

\author{
Florian Loeffl, Alexander Werner, Dominic Lakatos, Jens Reinecke, Sebastian Wolf, Robert Burger, \\ Thomas Gumpert, Florian Schmidt, Christian Ott, Markus Grebenstein, and Alin Albu-Schäffer
}

\begin{abstract}
Legged locomotion requires highly dynamic and efficient actuation as well as robust environment interaction. In the past years soft robots with elastic actuation have been investigated and their fitness for cyclic tasks and safe and robust environment interaction has been shown. To evaluate the benefits and drawbacks of series elastic actuation, variable impedance actuation as well as multi-articular elastic coupling in legged locomotion, we developed a two legged human size test-bed. These modular robotic legs give the possibility to evaluate and directly compare different elastic actuation concepts on a single system. Hopping and bipedal modal motion experiments where performed to proof the concept.
\end{abstract}

\section{INTRODUCTION}

Looking at the various robotic leg systems presented in literature, as we depict later on, we can find one-, twoand multi-legged systems. While the number of feet on one side increases the range of stable gaits and poses, it also increases the complexity and the footprint of the system. In single leg systems the gait pattern is restricted to one single gait: running; a two-legged system provides two gaits: walk and run. Multi-legged systems provide even more gaits: for quadrupeds for example walk, amble, trot, pace, gallop. We keep the complexity as low as possible but enable dynamic as well as quasi static locomotion patterns and focus in this work on two-legged systems. With respect to actuation, most humanoid systems use a more or less stiff motor-gearbox drive train. These systems provide accurate position controlled motion generation with high gain controllers. With torque controlled systems, providing software based compliance, a soft and safe environment interaction is possible. All these systems suffer from a limited shock robustness due to the limited compliance control bandwidth and the direct coupling of motor inertia to the link via the gearbox. In order to study inertia coupling effects as well as efficiency aspects, mass and size of a legged robot matters. Since the mass of a robotic system depends on its volume, it increases cubically with the size. The mass moment of inertia even depends by a power of five on the height because of the parallel-axes theorem. In small systems masses are quite low and also the mass moment of inertia is vanishingly small. Small forces and torques make these systems more sensitive to friction within their joints and drive trains. In our opinion a bigger system with considerable mass and inertia values will be more robust against model and parameter uncertainty and model assumptions. Therefore, we decided to focus for our

Robotic Mechatronic Center (RMC), Institute of Robotics and Mechatronics, German Aerospace Center (DLR), D-82234 Oberpfaffenhofen, Germany florian.loefflddr.de

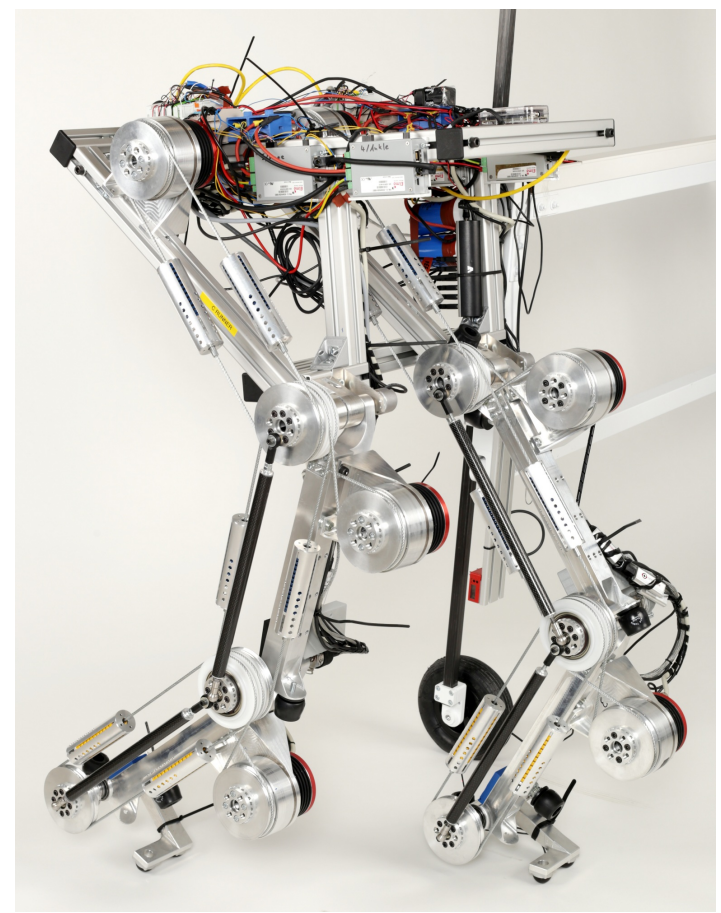

Fig. 1: The modular compliant leg test bench C-Runner

experimental study on a system of human size, allowing the comparison of different actuation principles.

There exist already several elastically actuated legged robots with either serial elastic drive concepts (SEA) e.g. Raibert hopper [1], ARL Monopod II [2], cCub [3], [4], BioBiped1 [5], MABEL [6], ATRIAS 1.0 [7] or variable impedance actuation (VIA) BiMASC [8], Blue [9]. Already in 1984 the Raibert hopper [1] was built with an air spring as elastic element in a single leg. The hip of the leg was moved by hydraulic actuators. Ahmadi and Buehler [2] used a helical spring in the leg and a medical latex in the hip joint as elastic elements. Additionally, the leg elongation and the hip angle could be actuated actively. In the work of Tsaganakis et al. [3] an asymmetric compliant antagonistic joint design is presented. It is a specialized antagonism with one high power branch protected by a torsional spring element for moving the joint and one high efficiency energy storage branch to save the energy in a spring element and to adjust the preloading in the elastic element. The stiffness cannot be changed in and it is a single joint, therefore, no bi-articularity is possible. For cCub [4] a compact SEA element was designed which works in a torsional manner, by compressing 
three springs with a wheel. With BioBiped1 [5] a robot leg is designed which mimics the muscles involved during dynamic motions of human legs. It uses simple springs for passive, mono and bi-articular actuation, but the focus is laid on the coupling structure rather than on verifying different compliant elements. Another bipedal robot is MABEL [6], a planar biped which has unidirectional leaf springs to store the huge amount of energy while running. It has no feet and no adjustable springs, but springs can be easily replaced. A parallel leg was used in ATRIAS [7], where carbon springs decouple the link and the actuator dynamically and are able to store the energy for efficient walking or running.

Variable stiffness can be found in BiMASC [8]. It uses joints which have actuators with the AMASC principle, enabling the robot to adjust leg stiffness to different gaits or ground surfaces. Another approach for variable stiffness with added variable damping can be found in Blue [9], which uses a modified version of the AwAs principle for varying the stiffness and a parallel motor for adjustable damping.

In contrast to an elastic element in series to the actuator, Hutter [10] used a bidirectional spring system differentially to include energy saving in the leg. The advantage is the possibility to place the heavy energy storing elements away from the fast moving leg, reducing the requirements of the actuator. But it loses partly the robustness feature because the gearbox is not completely decoupled from the impact.

With our leg test-bed we want to answer the question how much performance, compared to a standard SEA setup, can be gained introducing functional integration by multi-articular coupling, variable impedance actuation, or a SEA with parallel damper enhancing the control bandwidth.

In order to evaluate and compare these concepts as direct as possible, we decided to design a modular compliant leg test-bed with antropomorphic size and weight, which can be equipped with linear and nonlinear serial elastic actuators or variable impedance actuators, and also gives the possibility to implement multi-articular elastic couplings. Its wide range of possibilities enables us to compare the existing concepts and find the most suitable for efficient bipedal motion of a full size humanoid.

This work focuses on the design and implementation of the planar human size robotic leg test-bed. In Section IIB we will present our design considerations on the layout. Dimensions, kinematics, drives and instrumentation will be discussed. Section III addresses the physical implementation of the presented robot. We will present an approach for the design of elastic multi-articular couplings and show how this could be implemented on the C-Runner in Section IV. Preliminary proof of concept experiments are shown in Section V.

\section{CONCEPT AND SPECIFICATIONS}

\section{A. Specifications}

The list of specifications below gives an overview of the desired properties of the C-Runner testbed. We deduced the specifications from the following requirements to this robot.

1) Geometry and Kinematics

- 3 DoF planar mechanism (hip, knee, ankle)

- Human-like dimensions of the robotic leg

- Maximum speed $6 \mathrm{rad} / \mathrm{s}$ and torque $100 \mathrm{Nm}$

- Desired stiffness 200 - 800 Nm/rad

2) Actuation and Sensors

- Electromechanical actuation

- Joint torque measurement

- High resolution position measurement

- Ground force measurement

- Base state estimation

3) General Properties

- Good access to the system state by direct measurement

- Modularity: simple change of elastic elements or extension to bi-articularity

- Simple maintenance: good accessibility of all parts

\section{B. Concept}

The design concept for our C-Runner test-bed is based on a rigid body structure with two identical planar three-jointlegs attached to a common base frame. The four segments: base, thigh, shank and foot, are serially connected by the three rotational joints, hip, knee and ankle, to form a planar four link floating base kinematic structure. Each of the three segments base, thigh, and shank carries one servomotor. The drive axis of the motor is not coincident to the joint, to give space to the different compliance mechanisms. The transmission system should provide flexible connectivity between any joint and motor axis to allow motor to joint and joint to joint coupling. An agile controller development can be realised on a well observable system, providing measurements for all dimensions of interest. For best signal quality we try to measure relevant states directly. Also a simulation model of the robot matching quite closely the dynamic behaviour, has been developed.

\section{MeChatronic DESIGN AND DEVElopmEnt}

\section{A. Dimensions}

TABLE I: Joint workspaces

\begin{tabular}{|l|c|c|}
\hline Joint & Min. angle [rad] & Max. angle [rad] \\
\hline Hip & -2.09 & 0.54 \\
\hline Knee & 0 & 2.2 \\
\hline Ankle & -0.78 & 1.2 \\
\hline
\end{tabular}

The segment lengths are at human scale. They match the dimensions of an approximately $1,65 \mathrm{~m}$ tall human. Table II shows the segment lengths and masses. The workspace of the three joints is shown in table I 


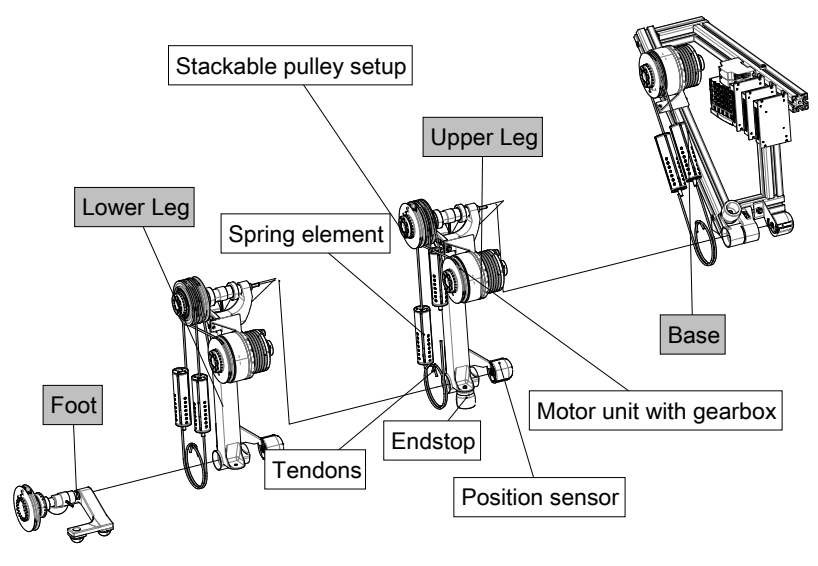

Fig. 2: Segments and joints. Each joint is equipped with a torque sensor and an absolute output angle sensor. Three segments have a servomotor driving one joint.

TABLE II: Segment heights and masses

\begin{tabular}{|l|c|c|}
\hline Segment & Height [m] & Mass [kg] \\
\hline Base & 0.276 & 24.96 \\
\hline Base width & 0.35 & \\
\hline Thigh & 0.40 & 5.46 \\
\hline Shank & 0.40 & 5.42 \\
\hline Foot & 0.07 & 1.31 \\
\hline Total & 1.15 & 49.34 \\
\hline
\end{tabular}

\section{B. Ground contact}

For ground interactions a $170 \mathrm{~mm}$ wide foot with two contact points was designed allowing the robot to balance. The contact points are silicone half domes giving the possibility to roll over during heel strike or ball push off. The silicone domes provide elasticity, damping and a high coefficient of friction. This results in shock absorbance and improved traction. Additionally, there are ground force sensors integrated into the silicone half domes, which are described in Sec. III-F.1.

\section{Compliant actuator modules}

A cable drive system was chosen to link the motor to the joints. This gives the highest freedom of design, since any joint can be driven by any motor with a wide range of transmission ratios steadily as well as non-uniformly. Joint couplings can be easily inserted. Every motor and joint axis has a spur gear hub providing an interface for an arbitrary combinable stack of pulleys. Figure 4 shows the spur gear hub with one pulley. Fixed pulleys with adjustable clamping fixture have been designed as well as free pulleys with ball bearings guiding the cable without transferring torque to the corresponding shaft. Robodrive ILM 85 motors have been chosen for the servo drives. These motors are combined with 80:1 Harmonic Drive gears to one compact drive unit. The motors are powered by Elmo DC Whistle servo controllers. Table III shows the motor specification and the expected performance after speed reduction in the gear.
TABLE III: Motor specifications

\begin{tabular}{|l|c|c|}
\hline & ILM85 & ILM85 + HD 80:1 \\
\hline Max. speed [rad/s] & 416 & 5.2 \\
\hline Peak torque [Nm] & 2,60 & 145 \\
\hline Continuos torque [Nm] & 1.43 & 80 \\
\hline Continuos power [W] & 450 & 315 \\
\hline Peak power [kW] & 1.1 & 0.754 \\
\hline
\end{tabular}

\section{Tendons}

As mentioned before, tendons are used for the drive train. They provide high peak forces and small weight. Steel tendons (CarlStahl 16022040) and Dyneema tendons (Ocean 7000, Sk 99) were chosen to be compared in detail.

Dyneema is a polymer tendon with very high load capacity due to extremely long molecular chains. The drawback of that chains is an intrinsic friction as described in [11], which results in torque losses when bending the tendon. Dyneema also allows a very small minimal bending radius. The main drawback is the creeping behaviour. We made an elongation test to find out if the creeping stops after holding a high load for a certain time. For this purpose, we used a commercial tensile testing machine (Zwick SN BZ1-MM14450.ZW01). In the first part of the settling experiment we drove the tendon five times from 0 to $4000 \mathrm{~N}$ to stress the tendon and then the tendon was held at $2000 \mathrm{~N}$ for 10 hours. As depicted in Figure 3, the tendon elongates less each cycle, but it is highly nonlinear and never stops creeping. Dyneema is not suitable for a bidirectional tendon actuation, since it is not able to hold the pretension and the mechanism we designed has no ability to readjust the loss of pretension.

For the alternative steel version a cable from Carlstahl was selected. It has 133 single braids grouped into 6 outer tendons and one core tendons of 19 braids with a total diameter of $4 \mathrm{~mm}$ and a maximum load of $9400 \mathrm{~N}$. Compared to Dyneema, steel tendons have no notable creeping and intrinsic friction [11]. The high number of braids allows rather small pulley diameter. Furthermore, it can be ordered directly with terminals that can hold the maximum load. For the test-bed we selected the steel version, since the noncreeping tendons are essential to keep the pre-tension.

\section{E. Springs}

To realise a serial elastic actuation we apply standard compression springs housed in a tube to get pull spring behavior. Each joint is equipped with two half pre-tensioned springs. This setup guarantees proper cable tension at any load. Three linear springs have been selected, providing the biggest spread of relevant stiffness for the available building space. For the experiments the spring stiffness, most suitable for the specific experiment where selected for each joint. We tested each spring type at our tensile testing machine (Zwick SN BZ1-MM14450.ZW01) to get better knowledge of the spring characteristics. The results are shown in Table IV.

\section{F. Electronics and software design}

1) Sensors: For good observability of the systems dynamic states, various sensors are placed in the robot. Table 


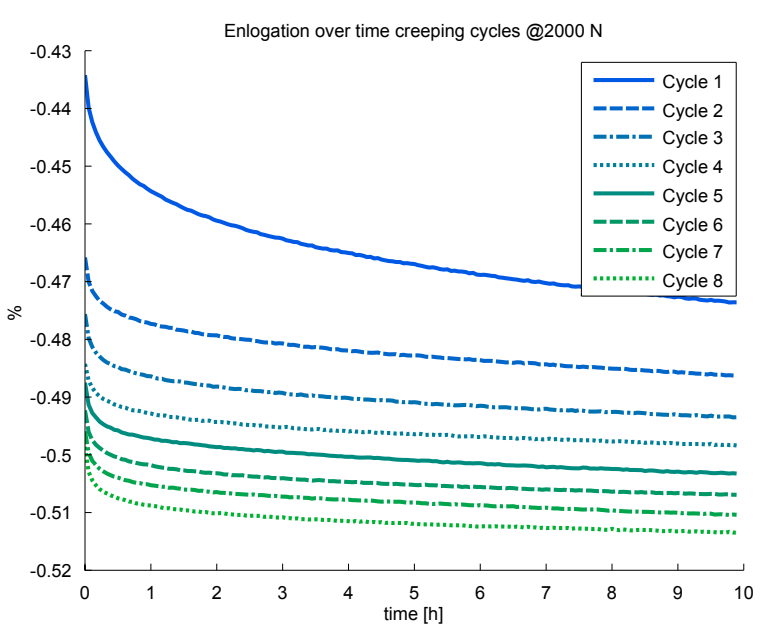

Fig. 3: Creeping test of the Dyneema tendons. Even after eight cycles the curve decreases.

TABLE IV: Spring specifications

\begin{tabular}{|l|c|c|c|}
\hline Type & $\begin{array}{c}\text { OR304- } \\
-616 \mathrm{D}\end{array}$ & $\begin{array}{c}\text { OR206- } \\
-618 \mathrm{D}\end{array}$ & $\begin{array}{c}\text { OR306- } \\
-616 \mathrm{D}\end{array}$ \\
\hline Nom. stiffness [Nm/rad] & 258 & 618 & 812.5 \\
\hline Nom. max. torque [Nm] & 105.25 & 189.1 & 207.2 \\
\hline Max. deflection [rad] & 0.408 & 0.306 & 0.255 \\
\hline Stiffness [Nm/rad] & 236.2 & 496.8 & 674.4 \\
\hline Linearity deviation [\%] & 3.8 & 3 & 5.2 \\
\hline Max. torque [Nm] & 111.75 & 200.3 & 200.2 \\
\hline Capacity [J] & 26.4 & 40.4 & 29 \\
\hline
\end{tabular}

$\mathrm{V}$ lists all integrated sensors, their types and their expected performance. Every joint output angle is measured by a 23 bit absolute angle encoder. We chose that high resolution to get good results for position as well as for the numerical derivation for speed and acceleration. The joint torques are measured by torque sensors with a range of $\pm 200 \mathrm{Nm}$. These analogue output sensors provide a $100 \mathrm{~Hz}$ bandwidth signal with $2 \%$ accuracy. The motor position is encoded with 18 bit, allowing a position resolution at the drive side of $80 * 18$ bit per revolution, which provides a well derivable signal, similar to the joint angle sensors. The motor winding temperature is observed by PT1000 temperature resistors to prevent overheating. Ground reaction forces are measured by three OptoForce sensors placed in the ground contact silicone domes. They give feedback about the reaction forces in three dimensions. The sensor in the heel has a range of $1500 \mathrm{~N}$ in vertical direction, the two parallel sensors in the ball of the foot have a maximal force of $600 \mathrm{~N}$ each. An inertial measurement unit (IMU) placed on the base segment provides 3D acceleration and rotational speed information for the base coordinates. The absolute height of the base is measured contact less by a laser distance sensor. Translational position and rotation angle are encoded by 23 bit absolute angle encoders placed under the center axis and into the base rotational joint.

2) Communication and bus: All communication on this robot takes place via two buses. The realtime network

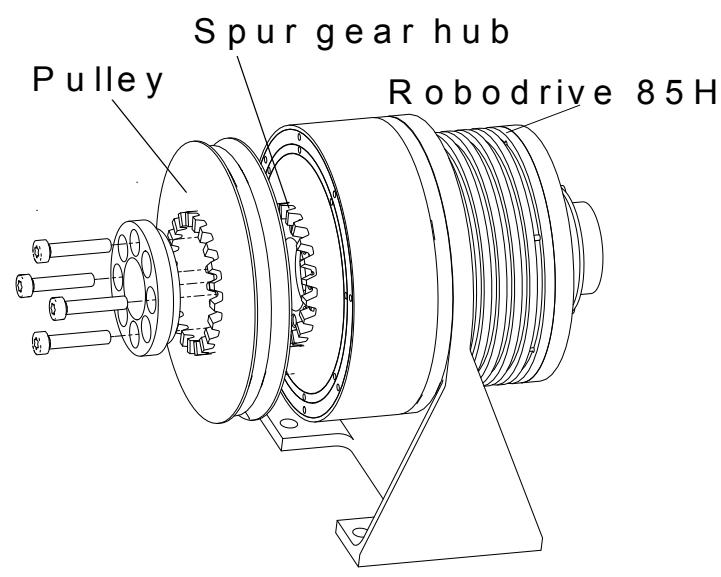

Fig. 4: Drive unit with fixed pulley and spur gear hub for modular drive train adaptability. More pulleys (free running or fixed) can be stapled onto the spur gear hub.

EtherCAT transmits the drive commands, as well as the position, torque and temperature measurements. An USB connection delivers the signals from the OptoForce ground contact sensors. A real time computer runs the EtherCAT bus at $1 \mathrm{kHz}$ and collects also the data from the USB.

3) Software and control: A runtime-configurable hardware abstraction framework called "Robot Kernel" developed at DLR-RMC provides an intra-module communication, a module synchronization mechanism, and access to the sensor data and motor-controller signals. This cross-platform software uses dynamically loadable device drivers, encapsulated into modules for a fast and flexible software implementation of the robot hardware. We use "Links and Nodes", to publish real-time control-loop signals with low latency using sharedmemory and condition variables to the compiled simulink model of the controller running on the real time computer. Via TCP the controller data is also accessible through "Links and Nodes" for the control PC. For more information refer also to the system setup of the Lightweight Rover Unit [12].

\section{Coupling}

\section{A. Coupling Design}

The rigid body dynamics of the articulated robot can be modelled by the differential equation

$$
\boldsymbol{M} \ddot{\boldsymbol{q}}+\boldsymbol{p}+\boldsymbol{d}=\boldsymbol{\tau}_{\mathrm{ext}}+\boldsymbol{\tau},
$$

where $\boldsymbol{M}(\boldsymbol{q})$ is the mass matrix, $\boldsymbol{p}(\boldsymbol{q}, \dot{\boldsymbol{q}})$ gravitational, centrifugal and Coriolis torques, $\boldsymbol{d}(\boldsymbol{q}, \dot{\boldsymbol{q}})$ damping torques, $\boldsymbol{q}(t)$ the joint angles and $\boldsymbol{\tau}(t)$ and $\boldsymbol{\tau}_{\text {ext }}(t)$ the joint and external torques.

For friction free linear spring coupling the joint and motor torque relationship is

$$
\left[\begin{array}{c}
-\boldsymbol{\tau} \\
-\boldsymbol{\tau}_{\mathrm{e}, \mathrm{m}}
\end{array}\right]=\boldsymbol{K}\left[\begin{array}{l}
\boldsymbol{q} \\
\boldsymbol{\theta}
\end{array}\right]=\left[\begin{array}{cc}
\boldsymbol{K}_{\mathrm{q}} & \boldsymbol{K}_{\theta \mathrm{q}} \\
\boldsymbol{K}_{\theta \mathrm{q}}^{T} & \boldsymbol{K}_{\theta}
\end{array}\right]\left[\begin{array}{l}
\boldsymbol{q} \\
\boldsymbol{\theta}
\end{array}\right] .
$$

Here $\tau_{\mathrm{e}, \mathrm{m}}(t)$ are the motor torques on the output of the gear, that is applied to the springs, $\boldsymbol{\theta}(t)$ the motor angles and $K K$ the stiffness matrix. 
TABLE V: Sensors and Measurements used in c-Runner with their expected performance

\begin{tabular}{|c|c|c|c|c|c|}
\hline Measurement & Symbol & Sensor type & Resolution & Accuracy & Bandwidth \\
\hline Joint angles [rad] & $\boldsymbol{q},(\dot{\boldsymbol{q}}, \ddot{\boldsymbol{q}})$ & Heidenhain EBI1023 & $7.49 \mathrm{e}-7$ & $2.9 \mathrm{e}-4$ & control rate $1 \mathrm{kHz}$ \\
\hline Joint torques $[\mathrm{Nm}]$ & $\tau$ & NCTE S-BB-RT & 0.03 & 10 & $100 \mathrm{~Hz}$ \\
\hline Motor positions [rad] & $\theta$ & Heidenhain ECI1135 & $3.00 \mathrm{e}-7$ & $7.27 \mathrm{e}-6$ & control rate $1 \mathrm{kHz}$ \\
\hline Hip acceleration $\left[\mathrm{m} / \mathrm{s}^{2}\right]$ & $\ddot{x}, \ddot{z}$ & ST LSM9DS1 & $6.1 \mathrm{e}-4$ & n.a. & $952 \mathrm{~Hz}$ \\
\hline Hip rotation speed $[\mathrm{rad} / \mathrm{s}]$ & $\dot{\phi}$ & ST LSM9DS1 & $1.53 \mathrm{e}-4$ & n.a. & $952 \mathrm{~Hz}$ \\
\hline Vertical base position [m] & $z$ & Leuze ODSL 9/C6-650-S12 & $1.98 \mathrm{e}-5$ & $6.5 \mathrm{e}-3$ & $500 \mathrm{~Hz}$ \\
\hline Horizontal base position [m] & $x$ & Heidenhain EBI1023 & $2.15 \mathrm{e}-6$ & $8.32 \mathrm{e}-4$ & control rate $1 \mathrm{kHz}$ \\
\hline Base orientation [rad] & $\phi$ & Heidenhain EBI1023 & $7.49 \mathrm{e}-7$ & $2.9 \mathrm{e}-4$ & control rate $1 \mathrm{kHz}$ \\
\hline Ground contact heel $[\mathrm{N}]$ & $W_{R}, W_{L}$ & OptoForce OMD-50-SA-1500N & 0.022 & 30 & $1 \mathrm{kHz}$ \\
\hline Ground contact bale [N] & $W_{R}, W_{L}$ & OptoForce OMD-30-SA-600N & 0.009 & 12 & $1 \mathrm{kHz}$ \\
\hline
\end{tabular}

From equation (2) we can deduct the necessary motor trajectory

$$
\boldsymbol{\theta}=-\boldsymbol{K}_{\theta \mathrm{q}}^{-1}\left(\boldsymbol{\tau}+\boldsymbol{K}_{\mathrm{q}} \boldsymbol{q}\right) .
$$

to track a desired task trajectory in $\boldsymbol{q}(t)$ and $\boldsymbol{\tau}(t)$ satisfying the rigid body dynamics (1).

To investigate our assumption bi-articular coupling could save motor power, we introduce the cost function

$$
J(\boldsymbol{K})=\alpha_{1} \frac{\sum_{n}^{i=1} \int\left|\dot{\theta}_{i}\right|}{\sum_{n}^{i=1} \int\left|\dot{q}_{i}\right|}+\alpha_{2} \frac{\sum_{n}^{i=1} \int\left|\tau_{\mathrm{e}, \mathrm{m} i}\right|}{\sum_{n}^{i=1} \int\left|\tau_{i}\right|}+\alpha_{3} \frac{\sum_{n}^{i=1} \int\left|\dot{\theta}_{i} \tau_{\mathrm{e}, \mathrm{m} i}\right|}{\sum_{n}^{i=1} \int \dot{q}_{i} \tau_{i}},
$$

summing up the distance the motor turns, normed by the distance the joint turns, the applied torques normed by the joint torques, and the spent energy of the motor, assuming braking also costs energy, normed by the work done by the joint. The three summands are weighted by $\boldsymbol{\alpha}=[4,1,1]$.

We generated a trajectory by simulating a hopping motion virtually constrained to pure vertical motion by the controller for running the optimisation

$$
\min _{\boldsymbol{K}_{\text {coupled }}}\left(J\left(\boldsymbol{K}_{\text {coupled }}\right)\right),
$$

where $\boldsymbol{K}_{\text {coupled }}$ has the structure

$$
\boldsymbol{K}_{\mathrm{q}, \text { coupled }}=\left[\begin{array}{ccc}
k_{1}+k_{12} & -k_{12} & 0 \\
-k_{12} & k_{2}+k_{12}+k_{23} & -k_{23} \\
0 & -k_{23} & k_{3}+k_{23}
\end{array}\right]
$$

and

$$
\boldsymbol{K}_{\theta}=-\boldsymbol{K}_{\theta \mathrm{q}}=\left[\begin{array}{ccc}
k_{1} & 0 & 0 \\
0 & k_{2} & 0 \\
0 & 0 & k_{3}
\end{array}\right]
$$

. This structure of $\boldsymbol{K}_{\text {coupled }}$ represents a leg with elastic couplings between base and thigh $\left(k_{12} \in[-1000,19000]\right)$, and between shank and foot $\left(k_{12} \in[-1000,19000]\right)$. Motor link couplings $k_{1}, k_{2}, k_{3}$ are choosen from the interval $[10,19000]$.

Table VI compares the maximum values of some measures for the desired jumping trajectory in $\boldsymbol{q}$ and $\boldsymbol{\theta}$ to the motor trajectory in $\boldsymbol{\tau}_{\mathrm{e}, \mathrm{m}}$ and $\boldsymbol{\theta}$ for the non coupled SEA setup

$$
\boldsymbol{K} ; \boldsymbol{K}_{\mathrm{q}}=\boldsymbol{K}_{\theta}=\left[\begin{array}{ccc}
200 & 0 & 0 \\
0 & 200 & 0 \\
0 & 0 & 200
\end{array}\right]
$$

TABLE VI: Simulated joint and motor torques for standard SEA setup and optimised bi-articular coupled C-Runner.

\begin{tabular}{|l|c|c|c|}
\hline $\begin{array}{l}\text { Measurement } \\
\text { Setup }\end{array}$ & Joint loads & $\begin{array}{c}\text { Motor loads } \\
\boldsymbol{K}\end{array}$ & $\begin{array}{c}\text { Motor loads } \\
\boldsymbol{K}_{\text {coupled }}\end{array}$ \\
\hline \hline Peak torque hip [Nm] & 44.44 & 44.44 & 59.83 \\
\hline Peak torque knee [Nm] & 199.20 & 199.20 & 163.32 \\
\hline Peak torque ankle [Nm] & 35.82 & 35.00 & 34.84 \\
\hline \hline Max speed hip [rad/s] & 3.54 & 3.33 & 3.26 \\
\hline Max speed knee [rad/s] & 4.16 & 4.85 & 4.02 \\
\hline Max speed ankle [rad/s] & 7.14 & 7.21 & 7.06 \\
\hline \hline Peak power hip [W] & 50.63 & 52.76 & 47.99 \\
\hline Peak power knee [W] & 658.04 & 798.04 & 442.56 \\
\hline Peak power ankle [W] & 113.34 & 113.57 & 148.95 \\
\hline
\end{tabular}

which was the base for the simulation, and the bi-articular coupled setup with the optimised

$$
\begin{aligned}
\boldsymbol{K}_{\text {coupled }} ; \boldsymbol{K}_{q} & =\left[\begin{array}{ccc}
1241 & -67 & 0 \\
-67 & 2212 & -18 \\
0 & -18 & 869
\end{array}\right] \\
\boldsymbol{K}_{\theta} & =\left[\begin{array}{ccc}
1308 & 0 & 0 \\
0 & 2296 & 0 \\
0 & 0 & 887
\end{array}\right] .
\end{aligned}
$$

This evaluation shows that by intelligent coupling, the motor peak power can be lowered under the joint peak power, leading to a more steady motor stress, and consequently allowing the use of smaller motors.

\section{B. Coupling Implementation}

The modular concept of C-Runner allows to implement both, the non coupled setup and the coupled one. Figure 5a illustrates the cable routing for the SEA case, connecting each servomotor through two pre-tensioned springs to the corresponding joint. The stiffness values of each antagonistic spring add up to the desired $k_{i}$. Any relative movement of $\boldsymbol{q}$ and $\boldsymbol{\theta}$ will tension one spring, and expand the other. That way torque can be transferred from motor to joint. In the coupled example Figure 5b, the spring setup is not symmetric and pre-tensioned, so the motor needs to tension the spring and always hold the pretension. The hip actuation remains more or less the same. In the knee the extending cable is attached to the motor, and the bending one winds around the hip axis and is fixed to the base frame. Same for the ankle joint, where the toe lifting cable is attached to the motor, and the antagonist winds around the knee axis, being attached to the upper leg. 


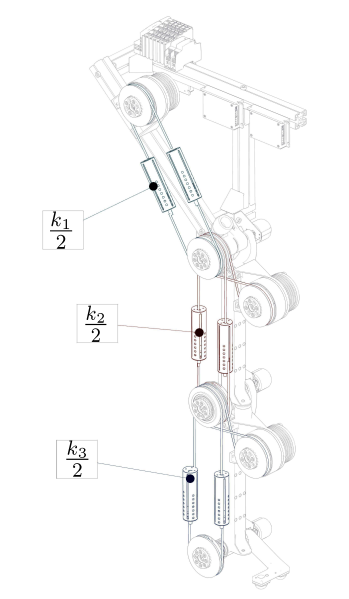

(a) Cablerouting for SEA setup $(\boldsymbol{K})$

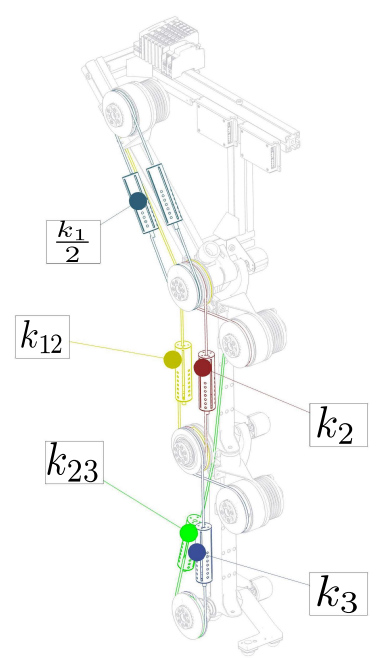

(b) Cablerouting for bi-articular coupled setup ( $\left.\boldsymbol{K}_{\text {coupled }}\right)$

Fig. 5: The modular concept allows direct motor joint connection as well as a bi-articular coupling.

\section{EXPERIMENTS}

The DLR C-Runner is intended to perform natural and efficient dynamic motions. Therefore, we provide experiments on a single leg and a bipedal setup showing how these natural motions can be achieved and validating the proposed energy efficiency of the mechatronic design.

\section{A. Controller}

The control method considered here can be used to induce a limit cycle for jumping on the spot. It is a simplified version of the controller introduced in [13]. The basic control principle exploiting the intrinsic mechanical properties of the plant is detailed in [14].

The controller switches the motor position

$$
\begin{aligned}
\boldsymbol{\theta} & =\boldsymbol{\theta}_{0}+\boldsymbol{w} \Delta \theta_{\mathrm{z}}\left(\tau_{\mathrm{z}}\right), \\
\Delta \theta_{\mathrm{z}}\left(\tau_{\mathrm{z}}\right) & = \begin{cases}\operatorname{sign}\left(\tau_{\mathrm{z}}\right) \hat{\theta}_{\mathrm{z}} & \text { if }\left|\tau_{\mathrm{z}}\right|>\epsilon_{\tau_{\mathrm{z}}} \\
0 & \text { otherwise }\end{cases}
\end{aligned}
$$

w.r.t. the equilibrium position $\boldsymbol{\theta}_{0}$. It is triggered by the generalized spring force

$$
\tau_{\mathrm{z}}=\boldsymbol{w}^{T} \boldsymbol{K}(\boldsymbol{\theta}-\boldsymbol{q})
$$

acting in the direction of the oscillation mode of the system specified by the modal weights $\boldsymbol{w}$. Thereby, $\epsilon_{\tau_{z}}$ is the torque threshold and $\hat{\theta}_{\mathrm{z}}$ is the switching amplitude.

Note that the controller parameter $\boldsymbol{\theta}_{0}$ can be tuned based on geometrical considerations. The modal weights $\boldsymbol{w}$ can be adapted by extracting the major principal component of the joint motion $\boldsymbol{q}(t)$ as explained in detail in [13]. The threshold $\epsilon_{\tau_{z}}$ is related to the amount of energy stored in the spring and the switching amplitude $\hat{\theta}_{\mathrm{z}}$ determines the amount of energy induced to the system. A detailed description of the basic control approach can be found in [14].

\section{B. Hopping}

The goal was to validate the capability of the human-sizeand-weight compliant leg to lift off its own body in a hopping motion. Therefore, we conducted jumping experiments with one leg on a vertical guide. Please note that with the given mechanical peak power of the actuators and the friction and damping losses of the HD gear-boxes, this is only possible due to energy storage in the real springs, which enable an increase of the retrievable power at the links, and a control algorithm which exploits this property.
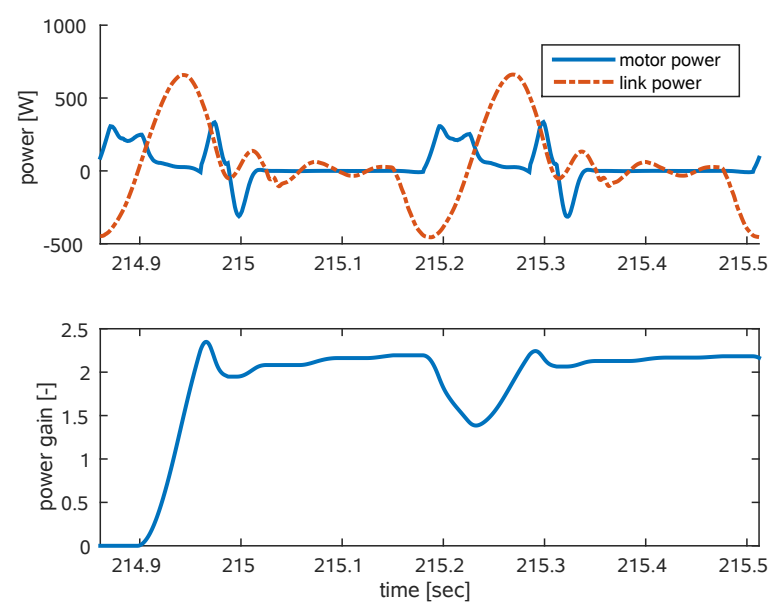

Fig. 7: Comparison of motor and link power of two steadystate jumping cycles.

The jumping motion has been initiated by a manually triggered switching of $\Delta \theta_{\mathrm{z}}=0 \rightarrow \hat{\theta}_{\mathrm{z}}$ and evolved to a periodic jumping motion. This already shows that the system is able to lift of its own body weight with energy exclusively inputed by the motors. The jumping motion can be also seen in the video attached to this paper.

The energy efficiency of the system is shown by comparing the time evolution of the motor-side power $P_{\mathrm{m}}(t)=\dot{\boldsymbol{\theta}}(t)^{T} \boldsymbol{\tau}_{\mathrm{m}}(t)$ and link-side power $P_{\mathrm{l}}(t)=$ $\dot{\boldsymbol{q}}(t)^{T} \boldsymbol{\tau}(t)$ as depicted in Figure 7. The upper plot in Figure 7 shows an increase in the link-side peak-power of $\max \left(P_{1}(t)\right) / \max \left(P_{\mathrm{m}}(t)\right) \approx 2$. To further quantify the energy efficiency of the system, we define the integral power gain

$$
\eta=\frac{\int_{t}^{t+T} \max \left(P_{1}(s), 0\right) \mathrm{d} s}{\int_{t}^{t+T} \max \left(P_{\mathrm{m}}(s), 0\right) \mathrm{d} s}
$$

where $T$ is an integer multiple of the oscillation period. This efficiency measure is evaluated in the lower plot of Figure 7. The value is $\eta \approx 2.2$ which indicates the energy efficiency of the system.

\section{Natural Bipedal Motion}

One of the key features of the C-Runner is the capability to display intrinsic mechanical oscillation modes, which can be exploited in the locomotion task, if they are excited 


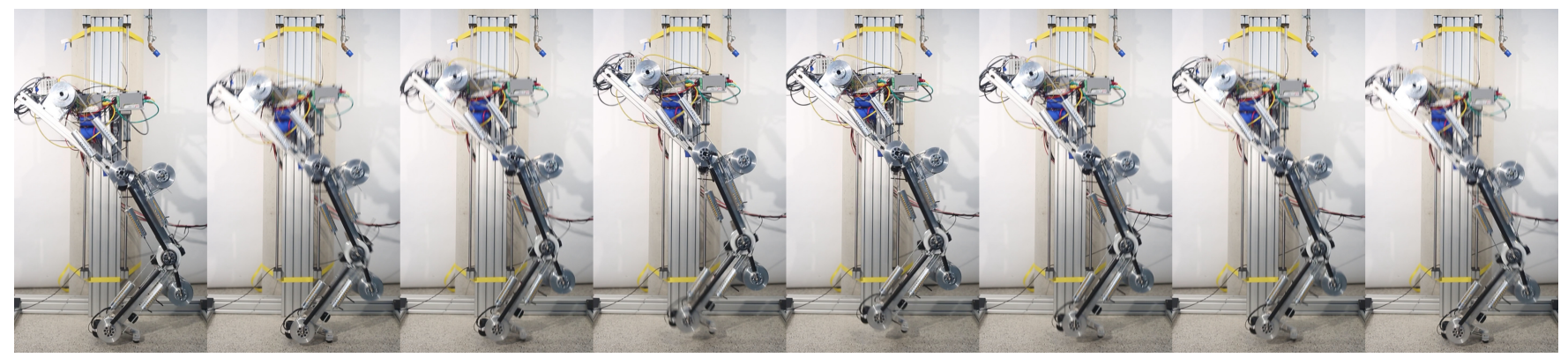

Fig. 6: C-Runner jumping on a spot as a proof of the ability to jump dynamically, while storing elastic energy in the springs.

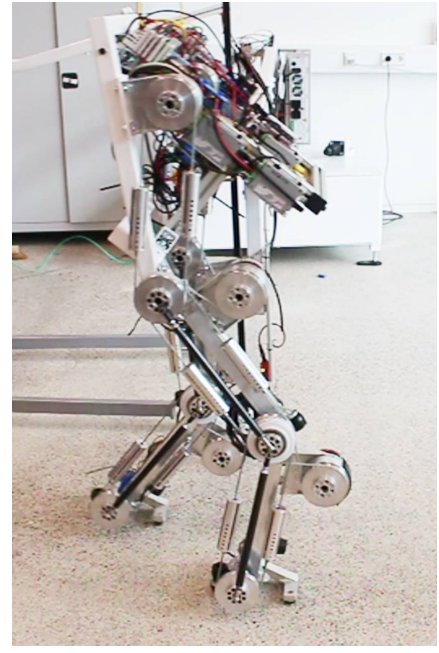

Fig. 8: Initial configuration of the natural motion experiment.

appropriately. The experiment described in the following first identifies and then excites such a dominant oscillation mode of the C-Runner hardware, while the latter is achieved using the control approach of section V-A.

To identify the dominant oscillation mode, i. e., the modal weights $\boldsymbol{w}$ as introduced in section V-A, the robot was controlled to maintain a certain motor configuration and placed on the ground such that the trunk was statically balanced (see, Figure 8). Then, natural oscillations were excited manually by pushing and releasing the base of the robot. Thereby, the motion of the joints was recorded and the zero mean data fed to a principal component analysis ${ }^{1}$. The major principal component of the oscillatory motion has been chosen as modal weights $\boldsymbol{w}$. Finally, the estimated eigenvector has been used in the controller of section V-A to excite and sustain a limit cycle (see, Figure 9) which can be used to initiate a step (see, the attached video).

This validates that the C-Runner displays an intrinsic mechanical oscillation mode which can be exploited to perform a locomotion task.

\footnotetext{
${ }^{1}$ Note that the principal components correspond to the eigenvectors of the data covariance matrix.
}

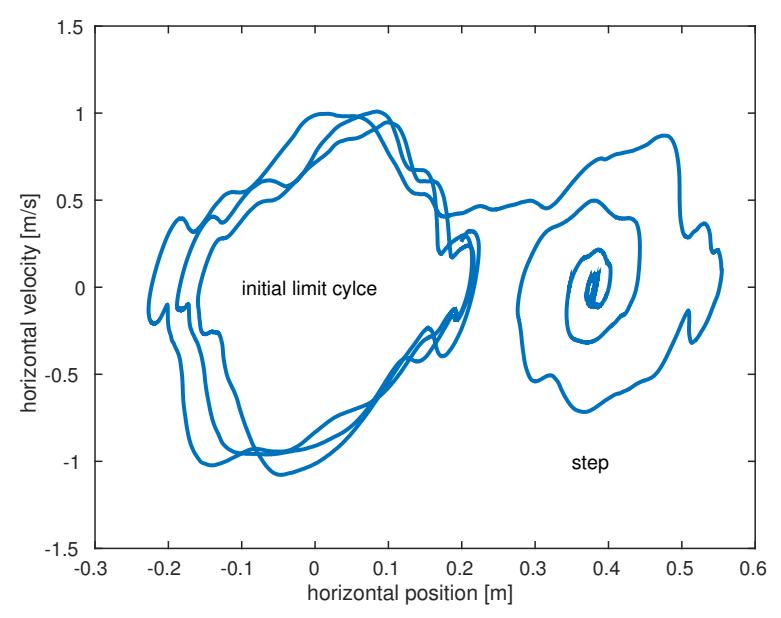

Fig. 9: Phase plot (trunk position vs. velocity) of the horizontal motion showing a limit cycle initiating a step. This experimental result validates that the C-Runner hardware displays an intrinsic mechanical oscillation mode which can be exploited for locomotion.

\section{CONCLUSION}

\section{A. Summary}

The paper presents the design and implementation of a modular two legged system. In the beginning we explained the need for modularity to study different compliance mechanisms, coupling, and control approaches on a single system. We expect a direct comparability of our future experimental results related to different actuation concepts obtained on this system. Our implementation is made up of servo motor modules non coincident to the joint axis. This allows us to design an arbitrary tendon drive train to the joints via pulley systems stacked upon the motor and joint axes by a spur gear hub. A very good observability via numerous sensors enables the use of model based control algorithms as well as delivering good experimental data. In first experiments applying a model free bang-bang controller to the system we could show the system jumping and it's ability to store energy physically in the springs. 


\section{B. Future work}

On this linear elastic SEA driven system we will study the model free bang-bang controller for jumping and running, as well as a model based limit cycle controller for compliant running. In addition, we produce optimal walking gait with trajectories generated with methods presented in [15]. We want to investigate the field of multi-articular elastic coupling by implementing the coupling proposed in section IV. After that a serial elastic element with adjustable damping will be integrated to increase the actuation bandwidth. Some experiments on a system as proposed in the work of Lakatos et al. [16] will follow.

\section{REFERENCES}

[1] M. H. Raibert, H. B. Brown, and M. Chepponis, "Experiments in balance with a 3D one-legged hopping machine," The International Journal of Robotics Research, vol. 3, no. 2, pp. 75-92, 1984.

[2] M. Ahmadi and M. Buehler, "The ARL monopod II running robot: Control and energetics," in Robotics and Automation, 1999. Proceedings. 1999 IEEE International Conference on, vol. 3. IEEE, 1999, pp. 1689-1694.

[3] N. G. Tsagarakis, S. Morfey, H. Dallali, G. A. Medrano-Cerda, and D. G. Caldwell, "An asymmetric compliant antagonistic joint design for high performance mobility," in Intelligent Robots and Systems (IROS), 2013 IEEE/RSJ International Conference on. IEEE, 2013, pp. 5512-5517.

[4] N. G. Tsagarakis, Z. Li, J. Saglia, and D. G. Caldwell, "The design of the lower body of the compliant humanoid robot cCub," in Robotics and Automation (ICRA), 2011 IEEE International Conference on. IEEE, 2011, pp. 2035-2040.

[5] K. Radkhah, C. Maufroy, M. Maus, D. Scholz, A. Seyfarth, and O. Von Stryk, "Concept and design of the BioBiped1 robot for human-like walking and running," International Journal of Humanoid Robotics, vol. 8, no. 03, pp. 439-458, 2011.

[6] J. Grizzle, J. Hurst, B. Morris, H.-W. Park, and K. Sreenath, "MABEL, a new robotic bipedal walker and runner," in American Control Conference, 2009. ACC'09. IEEE, 2009, pp. 2030-2036.
[7] J. A. Grimes and J. W. Hurst, "The design of ATRIAS 1.0 a unique monopod, hopping robot," in Proceedings of the 2012 International Conference on Climbing and Walking Robots and the Support Technologies for Mobile Machines, 2012, pp. 548-554.

[8] J. W. Hurst, J. E. Chestnutt, and A. A. Rizzi, "Design and philosophy of the BiMASC, a highly dynamic biped," in Robotics and Automation, 2007 IEEE International Conference on. IEEE, 2007, pp. 1863-1868.

[9] A. Enoch, A. Sutas, S. Nakaoka, and S. Vijayakumar, "Blue: A bipedal robot with variable stiffness and damping," in Humanoid Robots (Humanoids), 2012 12th IEEE-RAS International Conference on. IEEE, 2012, pp. 487-494.

[10] M. Hutter, C. D. Remy, M. A. Hoepflinger, and R. Siegwart, "High compliant series elastic actuation for the robotic leg ScarlETH," in Proc. of the International Conference on Climbing and Walking Robots (CLAWAR), no. EPFL-CONF-175826, 2011.

[11] J. Reinecke, M. Chalon, W. Friedl, and M. Grebenstein, "Guiding effects and friction modeling for tendon driven systems," in Robotics and Automation (ICRA), 2014 IEEE International Conference on. IEEE, 2014, pp. 6726-6732.

[12] A. Wedler, B. Rebele, J. Reill, M. Suppa, H. Hirschmüller, C. Brand, M. Schuster, B. Vodermayer, H. Gmeiner, A. Maier, et al., "LRUlightweight rover unit," in Proc. of the 13th Symposium on Advanced Space Technologies in Robotics and Automation (ASTRA), 2015.

[13] D. Lakatos, M. Görner, F. Petit, A. Dietrich, and A. Albu-Schäffer, "A modally adaptive control for multi-contact cyclic motions in compliantly actuated robotic systems," in Proc. IEEE/RSJ Int. Conf. on Intelligent Robots and Systems, 2013, pp. 5388-5395.

[14] D. Lakatos, F. Petit, and A. Albu-Schäffer, "Nonlinear oscillations for cyclic movements in human and robotic arms," IEEE Transactions on Robotics, vol. 30, no. 4, pp. 865-879, 2014.

[15] A. Werner, R. Lampariello, and C. Ott, "Trajectory optimization for walking robots with series elastic actuators," in Decision and Control (CDC), 2014 IEEE 53rd Annual Conference on. IEEE, 2014, pp. 2964-2970.

[16] D. Lakatos, C. Rode, A. Seyfarth, and A. Albu-Schäffer, "Design and control of compliantly actuated bipedal running robots: Concepts to exploit natural system dynamics," in Humanoid Robots (Humanoids), 2014 14th IEEE-RAS International Conference on, Nov 2014, pp. 930937. 